\title{
Large ovarian cysts assumed to be benign treated via laparoscopy
}

\author{
J. L. Herraiz $\operatorname{Roda}^{1}$ • J. A. Llueca Abella ${ }^{1}$ C. Catalá Masó ${ }^{1}$ • \\ Y. Maazouzi $^{1}$ - M. Colecha Morales ${ }^{1}$ - A. Serra Rubert ${ }^{1}$. \\ D. Piquer Simó ${ }^{1}$ - C. Oliva Martí ${ }^{1}$ - E. Calpe Gómez ${ }^{1}$
}

Received: 10 December 2014 / Accepted: 25 March 2015 /Published online: 7 April 2015

(C) Springer-Verlag Berlin Heidelberg 2015

\begin{abstract}
The aim of this study was to assess the feasibility and outcome of laparoscopic surgery in the management of large ovarian cysts in patients treated at a university hospital. Twelve patients with large (diameter $>10 \mathrm{~cm}$ ) ovarian cysts were managed laparoscopically from November 2009 to July 2014. The cystic masses were not associated with ascites or enlarged lymph nodes on ultrasound. Serum CA-125 levels were within the normal range $(35 \mathrm{U} / \mathrm{ml})$. Preoperative evaluation included history, clinical examination, sonographic images, and serum markers. The management of these ovarian cysts included aspiration, cystectomy, or salpingo-oophorectomy, depending on the patient's age, obstetric history, and desire for future fertility. Five patients presented with abdominal pain and two with abdominal distension and discomfort. In the five patients, the cyst was an incidental finding on a routine review. The average maximum diameter of the ovarian cysts was $25 \mathrm{~cm}$ (range 13-41 cm). The mean duration of the operation was $87 \mathrm{~min}$. The postoperative hospital stay was 14 days. No intraoperative complications occurred, and the hospital course of all patients was uncomplicated. In no case was laparoscopy converted to laparotomy. With proper patient selection, the size of an ovarian cyst is not necessarily a contraindication for laparoscopic surgery.
\end{abstract}

Keywords Laparoscopy · Large ovarian cyst · Minimally invasive surgery $\cdot$ Ovarian neoplasms

J. L. Herraiz Roda sgo.herraiz@gmail.com

1 Department of Obstetrics and Gynecology, General University Hospital of Castellón, Avenida Benicassim, 12004 Castellas, Spain

\section{Introduction}

Ovarian neoplasms are a common clinical problem, affecting females of all age groups. In the USA, it has been estimated that approximately $10 \%$ of the female population will undergo a surgical procedure for a suspected ovarian neoplasm during her lifetime [1].

Laparoscopy is considered as the gold standard approach to manage benign ovarian cysts. Treatment strategies of ovarian cysts are determined by the patient's age, menstrual status, symptoms, and the size and structure of the cyst [2]. The advantages of a laparoscopic approach over a laparotomy include better cosmetic results, less blood loss, less pain and analgesic requirement, faster recovery, and shorter hospitalization time [3].

A major factor affecting the gynecological surgeon's decision to perform a laparotomy is the size of the ovarian mass. The laparoscopic approach to large ovarian cysts extending to the umbilicus may be difficult because of the risk of cyst rupture and the small working space $[4,5]$. The laparoscopic management of very large ovarian cysts has been described [6-14], but most patients are managed by laparotomy.

The aim of this study was to evaluate the safety, effectiveness, and feasibility of laparoscopy in the management of ovarian cysts extending above the umbilicus. The results of the 12 patients with large ovarian cysts managed laparoscopically are reported herein.

\section{Materials and methods}

Twelve patients with very large ovarian cysts were included in the study. All of the patients underwent laparoscopy at the General University Hospital of Castellón, Spain, between November 2009 and July 2014. 


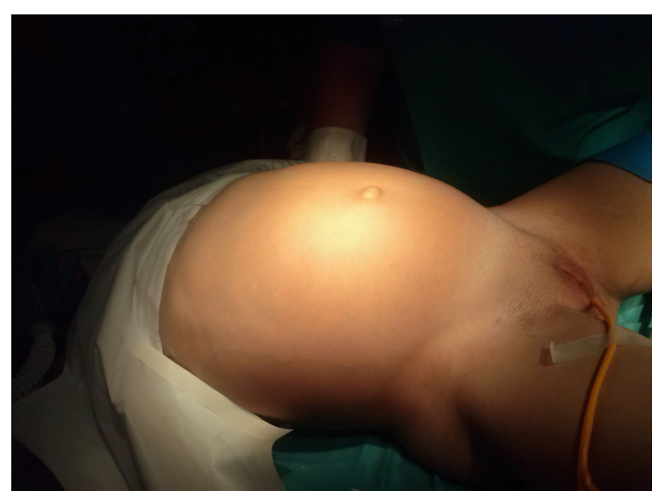

Fig. 1 Patient under general anesthesia

The cystic masses were not associated with ascites or enlarged lymph nodes on ultrasound. Serum CA-125 levels were within the normal range $(35 \mathrm{U} / \mathrm{ml})$ in all patients. Preoperative evaluation included history, clinical examination, sonographic images, and serum markers. No cases were excluded solely based on the cyst's size, only those suspected of malignant pathology. Therefore, out of all the cases, three patients with gigantic cysts did not undergo the laparoscopic procedure due to an elevated risk of malignancy. Two other cases were excluded due to high levels of serum markers and one due to the presence of ascites. Informed consent was obtained for possible conversion to laparotomy in case of technical difficulties

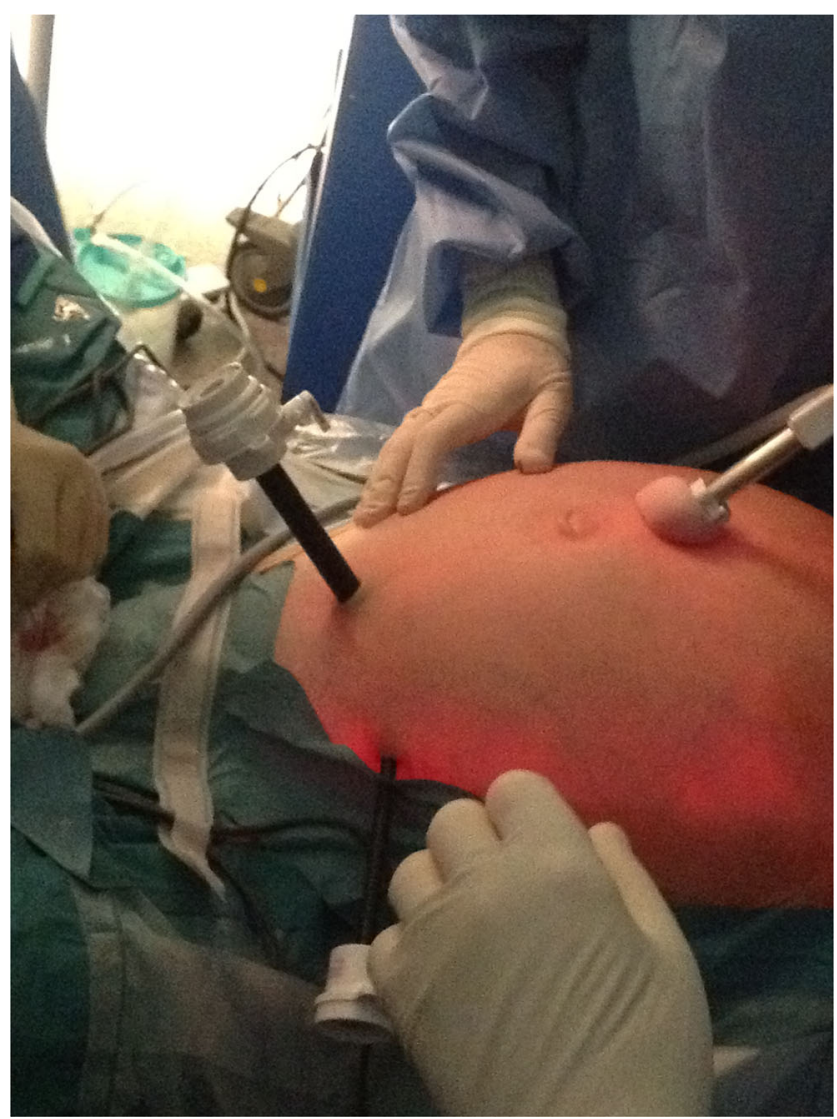

Fig. 2 The Hasson method (open-entry laparoscopic technique) in which three accessory trocars are inserted in the abdominal wall or an incidental finding of malignancy. All surgeries were performed with the patients under general anesthesia (Fig. 1).

The Hasson method (open-entry laparoscopic technique) was used to avoid puncturing the cyst prior to its intraoperative evaluation. The cyst wall was inspected prior to drainage. If there were no signs of malignancy, three additional trocars were inserted. The cyst was then drained under laparoscopic guidance using a suction irrigation device. If the size of the cyst was too large for this approach, a 3-cm umbilical incision was made that extended into the peritoneal cavity. An incision was made on the surface of the cyst, through which a Hasson trocar was then inserted. The cyst's content was then aspirated through the Hasson trocar in order to avoid spillage. Once the cyst was emptied, the Hasson trocar was removed and the cystic capsule was released into the abdominal cavity. The Hasson trocar was once again inserted in the abdominal wall in the usual manner. Three accessory trocars were then inserted, and the laparoscopic adnexectomy was performed using the usual method (Fig. 2). The cyst was decompressed, with careful attention to avoid spillage of its contents, prior to the placement of accessory trocars. Laparoscopic oophorectomy was then performed in the usual manner. The cystic mass was removed via a laparoscopic bag through the umbilical incision (Fig. 3). In no case was laparoscopy converted to laparotomy.

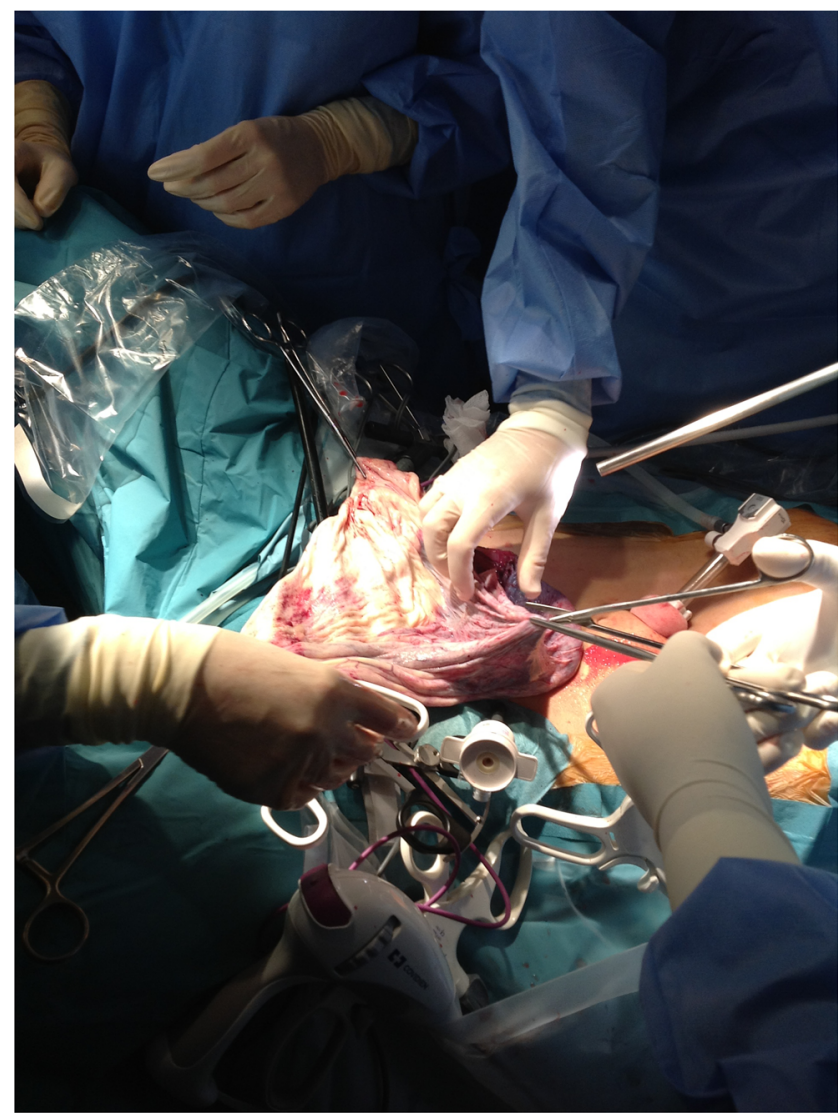

Fig. 3 Removal of a cystic mass 


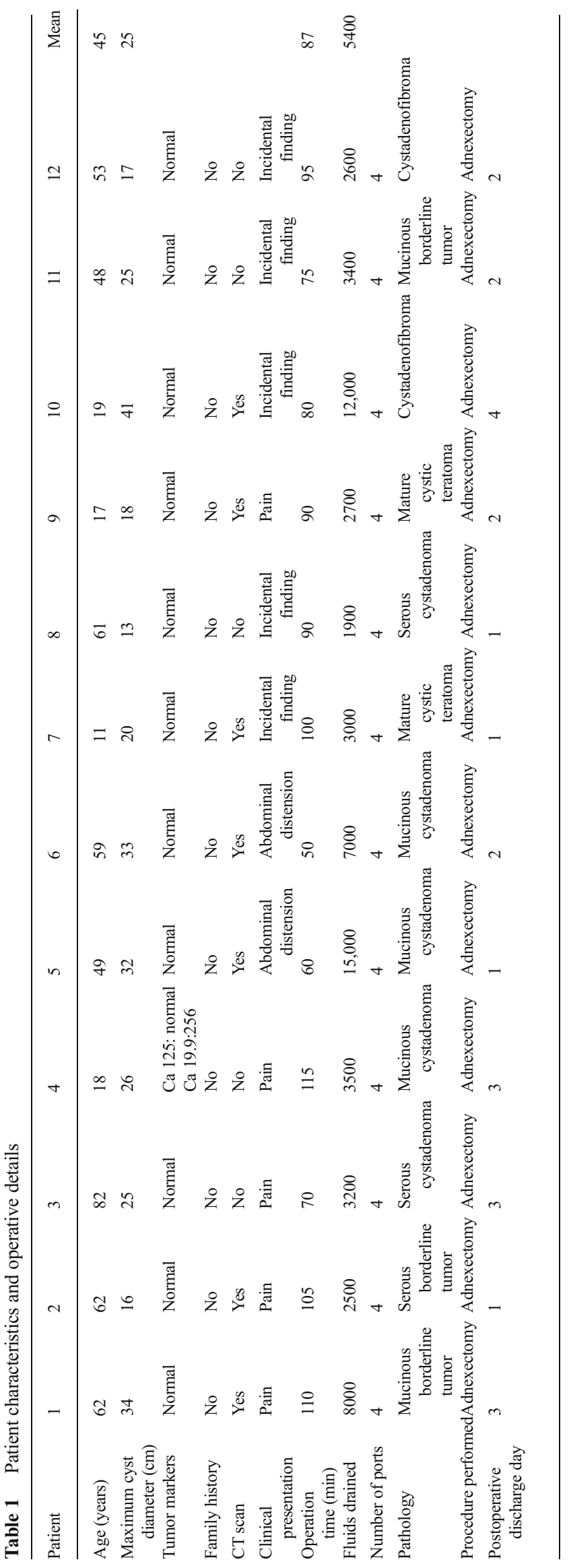




\section{Results}

All the patients had similar, non-specific presentations, including pain, abdominal distention, and discomfort. The mean age was 45 years (range 11-82 years) (Table 1). Three patients had previously undergone surgery for non-related causes. The family history was negative for ovarian cancer in all patients. The tumor markers CA-125, CA-19.9, and carcinoma embryonic antigen were normal in all patients, except in one patient with a CA-19.9 of 256 (Table 1). Transabdominal ultrasound scans performed in the 12 patients revealed a large unilocular cyst, some with fine septations, but no solid components or ascites. At our institution, neither computed tomography (CT) nor magnetic resonance imaging (MRI) is performed if the ultrasound findings are highly suggestive of a benign cystthat is, a unilocular cyst with no solid areas or thick septations and no ascites. However, some patients were referred with a CT scan (Fig. 4) and one with MRI. The mean size of the cysts as measured by preoperative ultrasound scans was $25 \mathrm{~cm}$ (range 13-41 cm).

The mean operative time was $87 \mathrm{~min}$ (range 50-115 min). The mean volume of fluid drained from the cysts was $5400 \mathrm{~mL}$ (range 1900-15,000 mL). Adnexectomy was performed in all patients. Histopathology revealed serous cystadenoma in two patients, mucinous cystadenoma in three, mature cystic teratoma in two, cystadenofibroma in two, serous borderline tumor in one, and mucinous borderline tumor in two.

Four trocars were used in each procedure, which were well tolerated by the 12 patients. There were no intraoperative or postoperative complications. All patients were discharged the next 1 to 4 days postoperatively.

\section{Discussion}

Very large ovarian cysts are traditionally managed using a full midline laparotomy [15]. Minimally invasive surgical techniques have been applied, but only a few cases have been reported. All reported techniques include decompression of the cyst to provide an adequate working space, facilitate manipulation of the cyst, and prevent inadvertent perforation and spillage.

Fifteen adult patients with giant $(>10 \mathrm{~cm})$ ovarian cysts as described by Salem underwent laparoscopic procedures. All of the cysts were benign, and the cyst fluid was aspirated after puncturing the cyst wall, after which the cyst was removed as usual. No conversions or other complications were recorded. Nine of the cysts were mucinous, and six were serous cystadenomas [16].

Other authors suggest drainage of these cysts via a minilaparotomy to allow for a more controlled approach to minimizing spillage than is possible with percutaneous

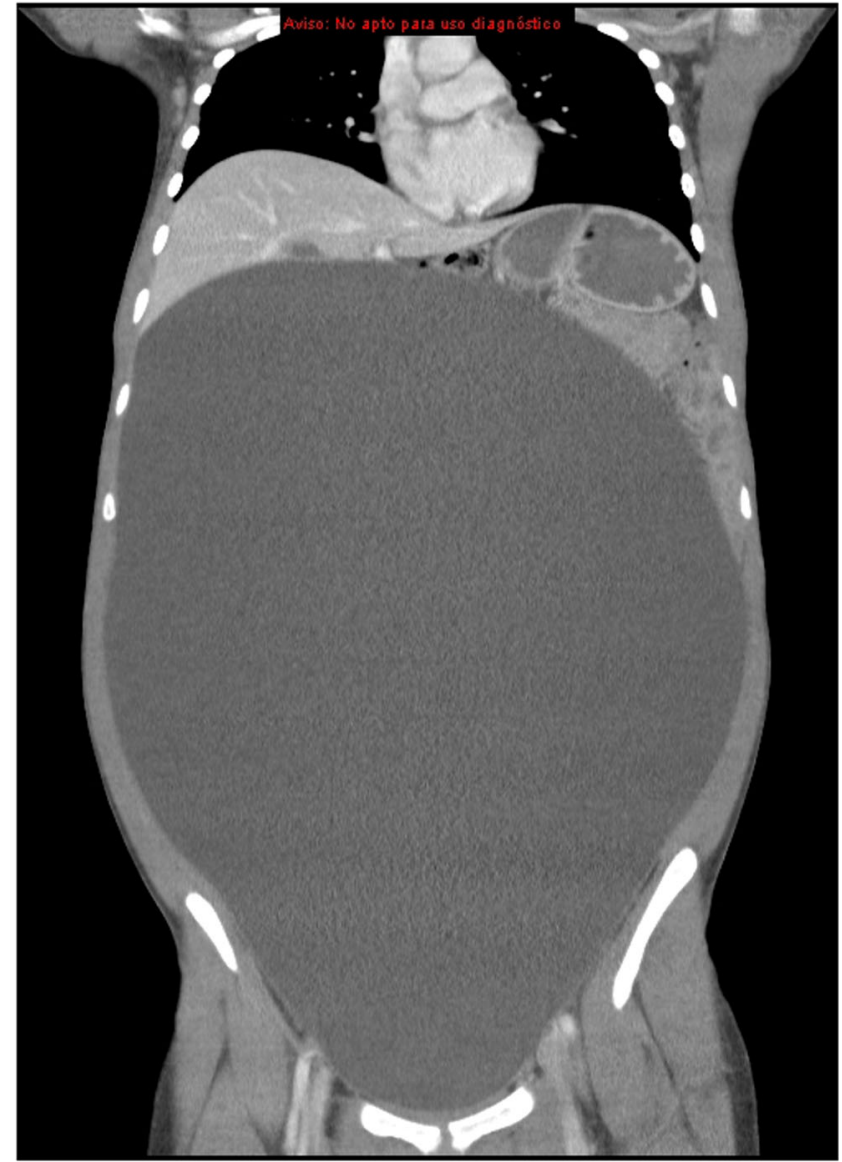

Fig. 4 CT scan of a patient with a large unilocular cyst

techniques; in addition, prelaparoscopic decompression is necessary to allow the establishment of a pneumoperitoneum for highly voluminous cysts [17].

Giant ovarian cysts can be drained before the laparoscopic approach to establish sufficient working space. Nagele [6] drained a large ovarian cyst with a Veress needle under ultrasonographic guidance before laparoscopy. Cevrioglu [18] performed a laparoscopic cyst excision after ultrasound-guided drainage with a spinal needle in a patient with a giant paraovarian cyst.

The use of laparoscopy in the management of ovarian cysts is determined by patient factors, including a history of previous abdominal surgery and premorbid conditions. However, this approach should provide all the benefits typically associated with laparoscopic techniques, i.e., decreased blood loss, less pain, shorter hospital stay, and a significantly better cosmetic result $[5,17]$.

The use of a laparoscopic approach for ovarian cysts with suspicious features is controversial owing to concerns related to potential spillage of the cyst contents into the peritoneal cavity. Spillage of dermoid cyst material can lead to an extensive inflammatory reaction, resulting in the formation of peritoneal adhesions, while spillage from a mucinous cyst may 
result in pseudomyxoma peritonei [19]. In the case of a malignant cyst, spillage of its contents can result in the intraperitoneal dissemination of malignant cells and thereby advance the stage of the disease [20].

It is uncommon to encounter an unexpected malignant ovarian mass. Nezhat intraoperatively discovered only four ovarian cancers in 1011 surgically managed patients [21]. There is no established guideline on the optimal timing of rescheduling the staging operation. A complete management plan based on accurate staging is more beneficial to patients in terms of long-term survival than under-treatment due to poor or no staging [22].

The literature data on the prognostic significance of intraoperative or surgical spill in the case of a malignant cyst are conflicting. In a meta-analysis of the effect of intraoperative rupture of the ovarian capsule on prognosis, Kim et al. [23] screened 518 studies and selected nine retrospective studies comprising 2382 patients. They found that preoperative rupture increased the recurrence rate when compared with intraoperative rupture (hazard ratio, 2.63; $95 \%$ confidence interval, 1.11-6.20). Patients with preoperative rupture had a poorer overall survival than those with no or intraoperative rupture.

Animal studies have shown that laparoscopy may accelerate the dissemination of malignant cells [24], but this has yet to be proven in humans. Childers et al. [25] commented that laparoscopy itself is not the cause of the problem for these patients and that surgical mismanagement can occur with any surgical approach.

\section{Conclusions}

With proper patient selection, minimally invasive surgery is a feasible and safe treatment of large ovarian cysts, demonstrating that size is not necessarily a consideration in the laparoscopic management of very large ovarian cysts. When performed by experienced endoscopic surgeons, laparoscopy may decrease the rate of unnecessary laparotomies for benign cysts.

Informed consent was obtained from all patients for being included in the study.

Conflict of interest On behalf of all authors, the corresponding author states that there is no conflict of interest.

\footnotetext{
Author's contribution JL Herraiz was responsible for project development, data collection, and manuscript writing; Y Maazouzi for manuscript writing/editing; A Llueca for protocol development; C Catala, M Colecha, D Piquer, A Serra, and C Oliva for data collection; and E Calpe for protocol development.
}

\section{References}

1. Hilger WE, Magriña JF, Magtibay PM (2006) Laparoscopic management of the adnexal mass. Clin Obstet Gynecol 49:535-548

2. Helmrath MA, Shin CE, Warner BW (1998) Ovarian cysts in the pediatric population. Semin Pediatr Surg 7:19-28

3. Yuen PM, Yu KM, Yip SK et al (1997) A randomized prospective study of laparoscopy and laparotomy in the management of unending ovarian masses. Am J Obstet Gynecol 177:109-114

4. Knudsen UB, Tabor A, Mosgaard B et al (2004) Management of ovarian cysts. Acta Obstet Gynecol Scand 83:1012-1021

5. Ma KK, Tsui PZ, Wong WC et al (2004) Laparoscopic management of large ovarian cysts: more than cosmetic consideration. Hong Kong Med J 10:139-141

6. Nagele F, Magos AL (1996) Combined ultrasonographically guided drainage and laparoscopic excision of a large ovarian cyst. Am J Obstet Gynecol 175(5):1377-1378

7. Jeong EH, Kim HS, Ahn CS et al (1997) Successful laparoscopic removal of huge ovarian cysts. J Am Assoc Gynecol Laparosc 4: 609-614

8. Postma VA, Wegdam JA, Janssen IM (2002) Laparoscopic extirpation of a giant ovarian cyst. Surg Endosc 16(2):361

9. Sagiv R, Golan A, Glezerman M (2005) Laparoscopic management of extremely large ovarian cysts. Obstet Gynecol 105:1319-1322

10. Eltabbakh GH, Charboneau AM, Eltabbakh NG (2008) Laparoscopic surgery for large benign ovarian cysts. Gynecol Oncol 108(1):72-76

11. Ate O, Karakaya E, Hakgüder G, Olguner M, Seçil M, Akgür FM (2006) Laparoscopic excision of a giant ovarian cyst after ultrasound-guided drainage. J Pediatr Surg 41(10):e9-e11

12. Goh SM, Yam J, Loh SF, Wong A (2007) Minimal access approach to the management of large ovarian cysts. Surg Endosc Interv Techn 21(1):80-83

13. Yi SW (2012) Minimally invasive management of huge ovarian cysts by laparoscopic extracorporeal approach. Minim Invasive Ther Allied Technol 21(6):429-434

14. Lim S, Lee KB, Chon SJ, Park CY (2012) Is tumor size the limiting factor in a laparoscopic management for large ovarian cysts? Arch Gynecol Obstet 285(5):1227-1232

15. Westfall CT, Andrassy RJ (1982) Giant ovarian cyst: case report and review of differential diagnosis in adolescents. Clin Pediat (Phila) 21:228-230

16. Salem AFH (2002) Laparoscopic excision of large ovarian cysts. J Obstet Gynaecol Res 28:290-294

17. Dolan MS, Boulanger SC, Salameh JR (2006) Laparoscopic management of giant ovarian cyst. JSLS 10:252-256

18. Cevrioglu AS, Polat C, Fenkc V et al (2004) Laparoscopic management following ultrasonographic-guided drainage in a patient with giant paraovarian cyst. Surg Endosc 18:346

19. Morrow CP (1998) Tumors of the ovary: classification: the adnexal mass. In: Morrow CP, Curtin J (eds) Synopsis of gynecologic oncology. Churchill Livingstone, Philadelphia, pp 215-232

20. Berek J (2000) Epithelial ovarian cancer. In: Berek J, Hacker N (eds) Practical gynecologic oncology. Lippincott Williams \& Wilkins, Philadelphia, pp 457-522

21. Nezhat F, Nezhat C, Welander CE, Benigno B (1992) Four ovarian cancers diagnosed during laparoscopic management of 1011 women with adnexal masses. Am J Obstet Gynecol 167(3):790-796

22. Alvarez RD, Kilgore LC, Partridge EE, Austin JM, Shingleton HM (1993) Staging ovarian cancer diagnosed during laparoscopy: accuracy rather than immediacy. South Med J 86:1256-1258

23. Kim HS, Ahn JH, Chung HH, Kim JW, Park NH, Song YS et al (2013) Impact of intraoperative rupture of the ovarian capsule on prognosis in patients with early-stage epithelial ovarian cancer: a meta-analysis. Eur J Sug Oncol 39:279-289 
24. Jacobi CA, Sabat R, Bohm B, Zieren HU, Volk HD, Muller JM (1997) Pneumoperitoneum with carbon dioxide stimulates growth of malignant colonic cells. Surgery 121:72-78
25. Childers JM, Nasseri A, Surwit EA (1996) Laparoscopic management of suspicious adnexal masses. Am J Obstet Gynecol 175: $1451-1457$ 